\title{
Physical exercise and cognitive performance in the elderly: current perspectives
}

This article was published in the following Dove Press journal:

Clinical Interventions in Aging

17 December 2013

Number of times this article has been viewed

\author{
Neva J Kirk-Sanchez' \\ Ellen L McGough ${ }^{2}$ \\ 'Department of Physical Therapy, \\ University of Miami Miller School \\ of Medicine, Miami, FL, USA; \\ ${ }^{2}$ Department of Rehabilitation \\ Medicine, University of Washington, \\ Seattle, WA, USA
}

\begin{abstract}
In an aging population with increasing incidence of dementia and cognitive impairment, strategies are needed to slow age-related decline and reduce disease-related cognitive impairment in older adults. Physical exercise that targets modifiable risk factors and neuroprotective mechanisms may reduce declines in cognitive performance attributed to the normal aging process and protect against changes related to neurodegenerative diseases such as Alzheimer's disease and other types of dementia. In this review we summarize the role of exercise in neuroprotection and cognitive performance, and provide information related to implementation of physical exercise programs for older adults. Evidence from both animal and human studies supports the role of physical exercise in modifying metabolic, structural, and functional dimensions of the brain and preserving cognitive performance in older adults. The results of observational studies support a dose-dependent neuroprotective relationship between physical exercise and cognitive performance in older adults. Although some clinical trials of exercise interventions demonstrate positive effects of exercise on cognitive performance, other trials show minimal to no effect. Although further research is needed, physical exercise interventions aimed at improving brain health through neuroprotective mechanisms show promise for preserving cognitive performance. Exercise programs that are structured, individualized, higher intensity, longer duration, and multicomponent show promise for preserving cognitive performance in older adults.
\end{abstract}

Keywords: aging, neurodegeneration, dementia, brain, physical activity

\section{Introduction}

The US population of individuals aged 65 years or older is expected to more than double, from 43 million to 92 million, by the year $2060 .{ }^{1}$ The prevalence of dementia and other cognitive impairment is also expected to increase incrementally. Normal aging is accompanied by alterations in brain structure and function, and associated cognitive changes. Although declines in cognition attributed to the normal aging process are well documented, some of these changes may be related to neurodegenerative diseases such as Alzheimer's disease (AD) and other types of dementia. In those over 71 years of age, the prevalence of dementia in the US is estimated to be nearly $14 \%$, with $10 \%$ attributed to $\mathrm{AD} .^{2}$ The prevalence of dementia increases from $5 \%$ of those 71-79 years of age to $37 \%$ of those over $90 .^{2}$ The estimated prevalence of cognitive impairment not categorized as dementia, including mild cognitive impairment (MCI), is $22 \%$, with $12 \%$ of these cases progressing to dementia annually. ${ }^{3}$ Epidemiological studies report reduced risk for MCI and dementia in older adults who maintain higher levels of physical activity. As physical exercise is essential in maintaining physical
Correspondence: Neva J Kirk-Sanchez Department of Physical Therapy, University of Miami Miller School of Medicine, 5915 Ponce de Leon Blvd., 5th floor Coral Gables, FL 33 I46, Miami, FL, USA

Tel +305 2844535

Email nkirksanchez@miami.edu 
function and physiological health, it also appears to be critical in maintaining brain health and cognitive performance in older adults.

The human brain begins to atrophy in the third decade of life, and there is disproportionate age-related atrophy in the frontal, parietal, and temporal regions. ${ }^{4}$ Normal age-related changes in executive function include decline in tasks that involve attention-switching (multitasking), difficulty in instrumental activities of daily living, slower response times, reduced speed of information processing, and reduced inhibitory control. ${ }^{5}$ Executive functions rely heavily on the frontal cortex, and the volume and function of this brain region declines with normal human aging. ${ }^{5}$ Language comprehension, particularly for complex text, is reliant on working memory, which also declines with normal aging. ${ }^{5}$ The hallmark of MCI and AD is brain atrophy with a more profound effect seen in the hippocampus, a medial temporal lobe structure involved in memory formation and marked by early pathology in individuals with AD. ${ }^{6,7}$ In fact, brain atrophy in key regions appears to occur prior to detectable cognitive changes. ${ }^{8}$ Brain regions vulnerable to agerelated and disease-related atrophy have also demonstrated changes in structure and function in response to moderate aerobic exercise, ${ }^{9,10}$ suggesting that physical exercise may enhance brain health and mediate improvements in cognitive performance.

The purpose of this review is to summarize the evidence supporting the effects of physical exercise on cognitive performance, and to discuss clinical implications and implementation of exercise interventions aimed at improving brain health. The definition of exercise used for this review is consistent with that of the American College of Sports Medicine (ACSM) position paper on prescribing exercise for adults. ${ }^{11}$ Exercise implies intentional physical activity for improving health and fitness. In this review, we present evidence on relationships between physical exercise and cognitive performance derived from observational studies evaluating physical activity and randomized controlled trials (RCTs) that focused on specific exercise interventions. We also discuss potential mechanisms through which exercise may mediate improved brain health and cognitive performance.

\section{Potential mechanisms: physical exercise and cognitive performance}

Multiple physiologic mechanisms likely account for neuroprotective and neuroplastic effects of exercise on brain structures. Many cardiovascular risk factors are associated with cognitive performance and risk for cognitive decline. ${ }^{12}$
Exercise-mediated physiologic mechanisms include elevated neurotrophin levels, improved vascularization, facilitation of synaptogenesis, mediation of inflammation, and reduced disordered protein deposition. ${ }^{13,14}$

\section{Exercise and cardiovascular risk factors}

Increased risk for cognitive impairment has been linked to cardiovascular risk factors such as hypertension, dyslipidemia, metabolic syndrome, uncontrolled diabetes, hyperinsulinemia, and high levels of inflammatory markers, all of which are modifiable by increasing exercise levels. ${ }^{15}$ Reduction of cardiovascular risk factors and improved levels of fitness have been associated with better brain health and cognitive performance in older adults. However, the mechanisms by which this effect occurs are unclear. Exercise may mediate cardiovascular risk factors that are linked to cognition and cognitive decline, or it may play a more direct role in neuroplasticity.

Hypertension (HTN) is associated with reduced cognitive performance at midlife, ${ }^{16}$ and longitudinal studies report increased likelihood of MCI and dementia in later life in individuals with elevated midlife systolic blood pressure. ${ }^{17-19}$ Associations between HTN and poorer performance on tests of attention, visuospatial skills, perceptual skills, memory, learning, psychomotor abilities, and executive function have been reported in older adults. ${ }^{12}$ In the brain, HTN is accompanied by reduced cerebral blood flow and metabolism, particularly in the frontal and temporal lobes and subcortical regions, ${ }^{20}$ and has been associated with white matter disease and atrophy. ${ }^{21,22}$ Although the mechanism remains unclear, hippocampal neurofibrillary tangles have also been reported in nondemented hypertensive individuals. ${ }^{23}$ Taken together, these brain changes suggest that HTN is an underlying neuropathologic precursor to cognitive impairment and dementia. Nevertheless, management of HTN appears to reduce the risk for brain pathology and cognitive impairment. For instance, older men on hypertensive therapy have shown less white matter disease than subjects with HTN who are not on hypertensive therapy. ${ }^{22}$ In fact, men on antihypertensive therapies for 12 years had no more risk for cognitive decline than those who were normotensive. Therefore, longterm pharmacological management of HTN appears to have neuroprotective effects. ${ }^{24}$ Regular aerobic exercise, maintained throughout midlife and older age, may well provide a protective effect on brain health and cognitive performance through the prevention and management of HTN, and subsequent enhanced cerebral blood flow and reduced brain tissue pathology.

Aerobic exercise has also been shown to play an important role in the management of type 2 diabetes and 
elevated cholesterol. ${ }^{15}$ This has important clinical implications considering study findings that older adults with metabolic syndrome have a $23 \%$ increased risk for cognitive impairment for every unit increase in the number of abnormal factors, including high body mass index (BMI), elevated triglycerides, low high-density lipoprotein cholesterol (HDL-C), HTN, and hyperglycemia. ${ }^{25}$ The association between metabolic syndrome and cognitive decline is much stronger in those with high levels of inflammatory markers, interleukin-6 and C-reactive protein, ${ }^{26}$ further supporting the need to employ strategies to modify these risk factors. ${ }^{15}$ Older adults who exercise have lower levels of inflammatory markers such as C-reactive protein and interleukin- $6 .{ }^{27}$ Serum homocysteine is also associated with vascular dysfunction, and has been shown to decrease following 6 months of highor low-intensity resistance exercise in older adults. ${ }^{28}$

There also appears to be a relationship between insulin resistance, brain health, and cognitive performance in older adults. Elevated plasma insulin levels that characterize insulin resistance invoke synchronous increases of Beta amyloid and inflammatory agents. ${ }^{29}$ One small clinical trial $(\mathrm{n}=28)$ suggested that in individuals with glucose intolerance, aerobic exercise improved cardiorespiratory fitness and glucose tolerance and these changes were also associated with improvements in several cognitive domains when compared to a group that performed only stretching exercises. ${ }^{30}$ The favorable effects of exercise are likely exerted through multiple pathways known to be influenced by insulin resistance, including improved cardiovascular and cerebral vascular function, reduced inflammation, and enhanced insulindependent energy metabolism. ${ }^{29}$ The relationship between exercise and reduction of cardiovascular risk factors is well established. ${ }^{31}$ However, it is unclear which cardiovascular factors serve as mediators in the relationship between exercise and cognitive performance.

\section{Exercise and neurotrophic factors}

The majority of evidence related to the underlying mechanisms of physical exercise on cognitive performance has been derived from studies of aerobic exercise; however, resistance exercise may also influence cognitive performance, possibly through other mechanisms. Furthermore, evidence suggests that physical exercise might be particularly important for apolipoprotein $\mathrm{E} 4$ carriers who have a genetic predisposition for the development of dementia. ${ }^{14}$

Neurotrophins such as brain-derived neurotrophic factor (BDNF) and nerve growth factor have been shown to facilitate plasticity and enhance neurovasculature in selected regions of the brain, including the hippocampus..$^{32}$ Increased release of protective neurotrophins are associated with higher levels of exercise in animal and human studies, ${ }^{33,34}$ and these physiological effects may have a positive impact on cognitive function in the aging brain. ${ }^{32}$ Insulin-like growth factor (IGF-1) promotes neuronal growth, survival, and differentiation, ${ }^{35}$ and increased serum levels of IGF-1 have been reported in older adults after 6 months of moderate to high levels of resistance exercise. ${ }^{36}$ BDNF, known to play a role in regulating growth, maintenance, and survival of neurons in the adult brain, increases in the blood of younger adults who engage in short-term ${ }^{33}$ or long-term ${ }^{34}$ aerobic exercise. In subjects performing 3 months of endurance training, resting levels of BDNF increased almost fourfold. ${ }^{34}$ In some clinical trials, aerobic exercise seems to be related to increased levels of BDNF, but not IGF-1, although these results are inconsistent. ${ }^{37}$ For example, clinical trials of resistance exercise are more likely to show an effect of increased levels of IGF-1 but not BDNF. ${ }^{37}$ Most of these trials have been performed in populations of young adults, and the carryover to older adult populations is unknown. Although BDNF levels in the brain are difficult to determine in humans, BDNF rapidly crosses the blood-brain barrier. It is likely that the blood serum levels in humans approximate levels in BDNF levels in the brain, but evidence is conflicting and further research is needed in this area. ${ }^{37}$

In summary, physical exercise that contributes to the reduction of cardiovascular risk factors is positively associated with biomarkers of brain health and improved cognitive performance. By affecting abnormal protein deposition, increasing neurotrophic factors, improving cerebral blood flow, and decreasing systemic inflammation, physical exercise may protect against degenerative brain changes associated with aging and neurodegenerative disease.${ }^{14}$ Resistance exercise also appears to influence underlying neural mechanisms of cognitive health. Figure 1 illustrates this relationship and the mechanisms by which exercise may have an impact on brain health and cognitive function.

\section{The role of exercise in neuroprotection and neuroplasticity Animal studies}

Animal research offers compelling evidence related to the role of exercise in promoting neuroplasticity in the brain. In rodents, early research demonstrated that access to exercise equipment (running wheels) helped neurons to grow and strengthened the connections within systems involved in learning and memory. ${ }^{37,38}$ Exercise in mice is accompanied 


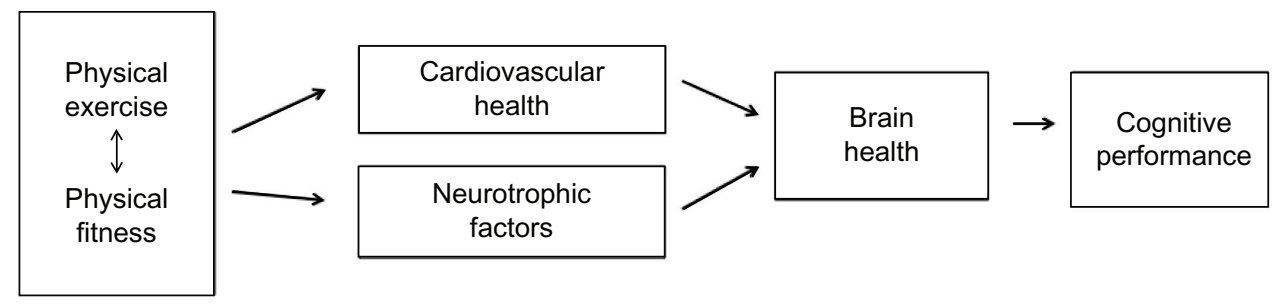

Figure I Physical exercise and cognitive performance in older adults: a theoretical model.

by increased levels of neurotrophic factors in the brain, such as BDNF, nerve growth factor, and IGF-1, which are associated with brain health. ${ }^{38}$ Specifically, Cotman and Berchtold demonstrated that rats who exercised showed increased gene expression of BDNF RNA (ribonucleic acid) in the hippocampus, as compared to control rats, and that the distance run per night was strongly correlated to BDNF levels in the hippocampus..$^{35}$ Rats that performed voluntary exercise on a running wheel showed enhanced learning and memory, as evidenced by improved performance in a water maze. These behavioral changes were also accompanied by decreased beta amyloid deposition in the exercising rats. ${ }^{39}$ Exercise also increases blood supply in the brain, thus facilitating nerve growth and nerve function. ${ }^{38}$ In animal models, these vascular changes appear to promote plasticity in the cortex, most profoundly in the hippocampus, and enhance growth and protection of neural structures. ${ }^{37}$

Mechanistic studies in rat models suggest that there is specificity of training that produces different types of changes in the brain, such as synaptogenesis and neurogenesis ${ }^{40}$ versus angiogenesis. ${ }^{41}$ In rats, a high-demand acrobatic training group was compared with forced exercise, voluntary exercise, and control group. Synaptogenesis occurred in the acrobatic group, suggesting formation of new circuits in response to a novel learning environment, while both exercise groups showed increased angiogenesis in the cerebellum. ${ }^{42}$ This result suggests some specificity of training toward precise mechanisms of improvement of brain health. ${ }^{10}$ The effect of exercise on brain health has also been studied using beta amyloid and Tau deposition in the brain as indicators. Although this effect has been difficult to assess in humans, in transgenic mice who overexpress the genes responsible for Tau and $\beta$-amyloid deposition in the brain, exercise appears to attenuate the deposition of these proteins in some studies. ${ }^{37}$ It is likely that a combination of exercise-mediated physiologic changes contributes to improved cognitive performance in animal studies. The effects of physical exercise on cognitive performance and potential physiological mechanisms observed in animal models may be translatable to humans.

\section{Cardiorespiratory fitness and brain health}

Research in older adults has demonstrated relationships between brain structure and function, cardiorespiratory fitness, and cognitive performance in relation to exercise levels. A Cochrane review of eleven RCTs examined the effectiveness of physical activity aimed at improving cardiorespiratory fitness on cognitive function and found an average of $14 \%$ increase in maximal oxygen consumption $\left(\mathrm{VO}_{2} \max \right)$ in the study subjects, who were healthy but sedentary older adults. ${ }^{43}$ Large effects of aerobic exercise on motor function and auditory attention, and moderate effects on cognitive speed and visual attention, were reported. ${ }^{43}$

Burns et al compared the relationship between cardiorespiratory fitness, as measured by peak oxygen consumption, and MRI-measured whole brain volume in subjects with mild $\mathrm{AD}$ versus no dementia. Whole brain volume was positively associated with cardiorespiratory fitness in the group with mild $\mathrm{AD}$, but not in the group without dementia. ${ }^{44}$ Similar results were found by Honea et al, who reported that cardiorespiratory fitness was related to both gray and white matter volume of the medial temporal and parietal regions in patients with early stage $\mathrm{AD}$, but not in nondemented subjects. ${ }^{45}$ These findings suggest that cardiorespiratory fitness may offer a protective effect on the brain by modifying AD-related changes in brain structure. ${ }^{45}$

Declines in tissue density in the frontal, parietal, and temporal cortices were examined in relation to age and cardiorespiratory fitness in 55 older adults with cognitive impairment. ${ }^{46}$ Brain regions that showed reduced gray matter and white matter volume with age were closely mirrored by brain regions that show preservation of volume with exercise. ${ }^{46}$ In 165 older adults without dementia, higher cardiorespiratory fitness levels were associated with preservation of hippocampal volume and better performance on a spatial memory task. Hippocampal volume was also 
positively associated with performance on the spatial memory task, supporting a mediating function of cardiorespiratory fitness. $^{47}$

Exercise and cardiorespiratory fitness may also influence cognitive performance by impacting brain activation ${ }^{48}$ and connectivity between brain regions. ${ }^{49}$ The relationship between brain activation and cardiovascular fitness was investigated cross-sectionally in a cohort of 41 older adults without cognitive impairment. Subjects with a higher estimated $\mathrm{VO}_{2}$ max had significantly greater brain activation in regions associated with executive control and less brain activation in areas postulated to interfere with this control..$^{48}$

Evidence from studies that measure brain connectivity using functional MRI suggests that the brains of older adults with higher levels of fitness are working more effectively than those who have lower levels of fitness. ${ }^{10}$ Some of these changes in connectivity, brain activity, and cognition might be attributable to angiogenesis and increased blood supply to the brain, but much evidence from animal studies, and to a lesser extent human studies, suggests that exercise also has an effect on levels of protective neurotrophins, which in turn may reinforce and protect neural connections. Modulation of brain health via cardiovascular health is evident in studies demonstrating a relationship between $\mathrm{VO}_{2}$ max and brain structure (reduced atrophy) and function (increased brain activation and connectivity). The positive effect of cardiorespiratory fitness on the brain is more profound in structures related to memory and executive function.

\section{Exercise and cognitive performance: observational studies}

People who are more active in midlife and late life have lower risk for global cognitive decline, ${ }^{50,51}$ and incident dementia. ${ }^{52-55}$ Longitudinal studies such as the HonoluluAsia Aging Study in men ${ }^{52,53}$ and the Nurses' Health Study in women ${ }^{50}$ have shown that higher levels of physical activity, including walking, are associated with a better cognitive function and lower risk of cognitive decline ${ }^{50}$ and dementia. ${ }^{52}$ The Honolulu-Asia Aging Study followed men from middle adulthood to late life to assess changes in physical activity and onset of dementia. Men $(n=2,257)$ were followed over three decades; those who walked less than 1 mile per day were at significantly higher risk (1.7-1.8 times) for developing dementia compared to men who walked more than 2 miles per day. ${ }^{52}$ Women in the Nurses' Health Study $(n=18,766)$ were followed for 10-15 years; for those aged 70-81 at follow-up, women who walked 90 minutes per week had global cognitive scores higher than those who walked less than 40 minutes per week. ${ }^{50}$

Bugg and Head investigated brain atrophy in two groups of older adults who performed levels of exercise below the group median and above the group median based on average metabolic equivalent (MET) hours per week over the previous 10 years..$^{56}$ The low exercise group performed an average of $0.63 \pm 74$ MET hours per week and the high exercise group performed an average of $7.8 \pm 3.9$ MET hours per week, the latter value being roughly equivalent to moderate exercise for 30 minutes five times per week. In both groups, age was correlated to brain atrophy in several areas of the brain, but in some subcortical areas, including the medial temporal lobe, age was correlated with brain volume in the low exercise group but not the high exercise group. ${ }^{56}$ Thus, in this cohort, total exercise volume seems to moderate the amount of atrophy in the medial temporal lobe, a key area for memory and executive function. Importantly, the exercise volume which appeared to be protective is similar to the amount of exercise recommended for all older adults. ${ }^{15}$

Although the optimal exercise dosage (intensity and duration) and type of exercise remain unclear, positive relationships between a higher dosage of exercise and cognitive health have been reported in aging adults. In a meta-analysis of 16 prospective cohort studies, the relative risk for dementia and $\mathrm{AD}$ were lowest in those with the highest level of physical activity, suggesting a protective effect of high levels of physical activity that reduces the risk of dementia by $28 \%$ and $\mathrm{AD}$ by $45 \% .^{55}$

Some longitudinal studies have demonstrated dosage effects of physical activity on brain health ${ }^{57}$ and cognitive decline..$^{51}$ The FINE study, utilizing surviving cohorts from seven countries $(n=295)$, described a dose response effect, where differences were based on physical activity dose over a 10 -year period. ${ }^{51}$ In this cohort, men who became less active over 10 years, as indicated by a decrease in activity of more than 60 minutes per day, had a 2.5 times higher risk for cognitive decline. Men whose physical activity intensity was reduced by 0.5 standard deviation (SD) or more over 10 years had a 3.6 times higher risk for cognitive decline. ${ }^{51} \mathrm{~A}$ similar finding related to exercise duration in women over the age of 65 was demonstrated by Yaffe et al from the Study of Osteoporotic Fractures cohort $(\mathrm{n}=9,704) .{ }^{54}$ Women who walked the fewest number of blocks per week had the highest odds of cognitive decline over 6-8 years, after adjusting for age and age-related factors..$^{54}$ These findings are in agreement with findings from the Cardiovascular Health Study, in which older adults $(n=299)$ were examined over a 9-year period. In this 
cohort, walking activity over a 1 -week period at baseline was predictive of greater gray matter volumes in the frontal, occipital, and medial temporal lobe 9 years later, even after controlling for baseline measures of white matter lesions and MRI infarcts. ${ }^{57} \mathrm{~A}$ relatively long distance at baseline (72 blocks/week) was necessary to detect differences 9 years later. Furthermore, greater gray matter volume in the brain was associated with reduced risk of cognitive impairment over the 9-year period. ${ }^{57}$

Two longitudinal studies have provided evidence that performing a variety of exercises or physical activities is beneficial. ${ }^{58,59}$ The Doetinchem Cohort Study examined healthy men and women $(n=1,927)$ at baseline and at 6 - and 11-year follow-ups, assessing the time spent (hours), the intensity (metabolic equivalents), and the variation (number of different activities) of physical activities performed. This study found that intensity and variation of physical activities was positively associated with processing speed, memory, mental flexibility, and overall cognitive function. ${ }^{58}$ Interestingly, the duration of time spent performing physical activities was not associated with cognitive performance. ${ }^{58}$ The Cardiovascular Health Cognition Study had similar findings, reporting that people in the highest quartile of energy expenditure had a 15\% lower risk of incident dementia after 5 years as compared to those in the lowest quartile, and those engaging in more than four different physical activities had only half the risk of incident dementia when compared to people engaging in one or no physical activities. ${ }^{59}$

In a cross-sectional study of men and women age 68-92 years $(n=122)$, physical activity and weekly energy expenditure were associated with better performance in executive function, as measured by the Stroop Color-Word test, after controlling for age, IQ, and education. ${ }^{60}$ The Mayo Clinic Study of Aging examined self-reported midlife and late-life exercise in a cohort of older adults $(n=1,324)$ who were categorized as having either no cognitive impairment or MCI. In this cohort, any frequency of moderate exercise in midlife or late life was associated with reduced odds of MCI. ${ }^{61}$

In summary, the results of cross-sectional and prospective longitudinal studies provide consistent and compelling evidence for a protective benefit against cognitive decline offered by higher levels of physical activity. Dose-response studies have not been conducted to determine the optimal level of exercise needed for cognitive improvement, but it appears that duration and variety of physical activities are important factors.

\section{Exercise and cognitive performance: intervention studies}

In the last decade, a number of intervention studies have investigated the effects of aerobic exercise, resistance exercise, and multicomponent interventions on cognitive function and brain health in adults with and without cognitive impairment. Although one meta-analysis of 29 RCTs concluded that adults performing aerobic exercise improved in attention and processing speed, executive function, and memory, ${ }^{62}$ other meta-analyses of 30 trials $^{63}$ and 23 trials $^{64}$ failed to demonstrate that physical exercise has a positive effect on cognitive performance.

Multicomponent exercise interventions, such as combined aerobic exercise and strength training programs, may have a larger effect on cognition than aerobic exercise alone. ${ }^{62}$ Meta-analysis by Smith et $\mathrm{al}^{62}$ also suggests that clinical trials of exercise for people with MCI may be associated with greater improvements in memory than trials for people without cognitive impairment; however, this has not been supported by other meta-analyses. ${ }^{65}$

\section{Aerobic exercise interventions}

Aerobic exercise RCTs in older adults have demonstrated effects on cognitive performance in conjunction with changes in regional brain volume, ${ }^{9,66}$ neurotrophin levels, ${ }^{66}$ and brain activation patterns. ${ }^{67}$ Colcombe et al ${ }^{9}$ compared a 6-month aerobic exercise program for sedentary communitydwelling adults to a control group that performed stretching and toning exercises. The group that performed aerobic exercise showed an increase in gray and white matter volume in prefrontal and temporal cortical regions, which are thought to be responsible for higher order attentional control and memory and are also prone to age-related atrophy. ${ }^{9}$ Although this research offers compelling evidence about the benefits of aerobic exercise on brain health, it is unknown whether changes in brain structure or function were accompanied by cognitive improvement in this cohort. Similarly, Erickson et al compared a group of sedentary nondemented older adults who participated in an aerobic exercise (walking) program versus a group who did only stretching exercises and showed that the aerobic exercise group had improved cardiovascular fitness with a $7.8 \%$ increase in $\mathrm{VO}_{2}$ max. ${ }^{66}$ The aerobic exercise group also demonstrated improvement in spatial memory, increased anterior hippocampal volume by $2 \%$, and increased levels of BDNF in the hippocampus. ${ }^{66}$

Changes in functional connectivity patterns were reported in a cohort of older adults with no cognitive impairment that engaged in an aerobic exercise program. Increased changes in 
activation in executive control regions and decreased changes in activation in other regions were found in subjects who performed the aerobic exercise intervention as compared to subjects engaged in a stretching and toning control group. ${ }^{67}$ Improved functional connectivity between regions prone to age-related disruption was seen after 12 months of moderate aerobic training, and this was associated with improvements in executive function. ${ }^{67,68}$ Furthermore, changes in functional connectivity were associated with changes in BDNF and other growth factors. ${ }^{68}$

Although less evidence is available for the effect of exercise interventions in people with cognitive impairments, in one study subjects $(n=138)$ participating in a 24 -week selfmonitored home-based walking program were compared to a control group of subjects who received education and usual care. Subjects who participated in the walking program showed modest improvement in global cognition as measured by the Alzheimer's Disease Assessment Scale - Cognitive Subscale. ${ }^{69}$ Another study showed that subjects $(n=29)$ with MCI who participated in 6 months of a high-intensity exercise program ( $75 \%-85 \%$ of heart rate reserve), as compared to those who only stretched, demonstrated improvements on tests of executive function; however, this effect was larger for women, with men only showing effects in one test of executive function..$^{70}$ Additionally, the exercise group showed changes in BDNF levels in women and IGF-1 levels in men. ${ }^{70}$ However, this study was limited by a very small sample size. ${ }^{70} \mathrm{~A}$ third study examined the effect of a 12-month group exercise program, which was offered 90 minutes per day, two times weekly, for older adults $(n=50)$ with MCI. Compared to an education-only control group, the participants in the exercise group showed better general cognitive function, immediate memory, and language ability. ${ }^{71}$ A fourth study in inactive community-dwelling older adults with memory complaints $(n=126)$ participating in a 12 -week, three times weekly intervention of aerobic exercise, mental exercise, or a combination of aerobic and mental exercise, reported significant improvements in a composite score of cognitive function with no evidence of difference between intervention and active control groups. These findings demonstrated improvement in cognitive performance after 12 weeks of either physical or mental activity; however, it is possible that the dosage of physical exercise was not sufficient to affect the maximal potential for cognitive performance via physical exercise. ${ }^{72}$

\section{Resistance exercise interventions}

Although few studies have examined the effects of resistance training on cognitive function, there is some evidence that resistance-only training has a positive effect. A 24-week study in sedentary older men $(n=63)$ without dementia ( $>23$ minimental state evaluation [MMSE]) compared a moderate- and high-intensity resistance exercise protocol group (upper and lower body resistance at $50 \%$ and $80 \%$, one repetition max) to a stretching control group. Both moderate- and high-intensity exercise groups showed improvements in several cognitive domains, and both exercise groups showed increased levels of IGF-1, thought to be related to preservation of cognitive function. ${ }^{36}$ A study of women $(n=155)$ examined the cognitive effects of a 12-month once- or twice-weekly resistance training program versus a control program of balance and toning exercises. Women who participated in a 12-month resistance exercise program one to two times weekly demonstrated improved performance on tests of executive function, including selective attention and conflict resolution. Improvements in cognitive performance in the resistance exercise group were present after 12 months of training, but not after 6 months of training, suggesting that a longer duration of resistance training is needed to bring about cognitive change. ${ }^{73}$ In another study, older women who engaged in a 6-month, twice-weekly resistance training versus a balance and toning control program significantly improved their performance on a test of response inhibition in conjunction with increased hemodynamic activity, as measured by functional magnetic resonance imaging, in brain regions commonly associated with response inhibition. This provides support that resistance exercise contributes to functional plasticity in brain regions associated with executive function, specifically response inhibition. ${ }^{74}$

In summary, positive effects of exercise on cognitive performance are demonstrated in some RCTs; however, other trials show minimal to no effect of exercise on cognition, and the results of meta-analyses are equivocal. Although further research is needed in this area, evidence supports the need for longer duration exercise interventions (aerobic and resistance) to show improvement in cognitive performance. Several meta-analyses suggest that at least 6 months of exercise is necessary to detect cognitive changes, although changes in brain function have been demonstrated in trials of shorter duration. ${ }^{10}$ At this time, we have insufficient evidence to determine whether specific exercise intensities have a differential effect on cognitive performance.

\section{Multicomponent exercise interventions}

The majority of exercise intervention trials studying cognitive effects focus on either aerobic or resistance training; few have investigated multicomponent exercise programs. 
In a study of 74 community-dwelling older adults, a homebased balance and strengthening program aimed at reducing falls also significantly improved executive function, specifically response inhibition, after 6 months. ${ }^{75}$ Tai Chi is another example of a multicomponent exercise that has potential to mediate the relationship between physical exercise and cognitive performance in older adults; however, current evidence is limited, and research is needed on exercise parameters and effectiveness in specific cognitive domains. ${ }^{76}$ In addition to multiple physical components, Tai Chi incorporates engagement across several cognitive domains. In a crosssectional study comparing cognitive performance in relation to self-reported participation in Tai Chi versus cardiovascular exercises, older adults completing both types of exercise demonstrated better memory performance than those who practiced one or the other or no exercise. ${ }^{77}$ Lam et al studied progression to clinical dementia over a 1-year period following a Tai Chi intervention $(n=171)$ versus a stretching and toning control group $(n=218) .{ }^{78}$ Although Tai Chi showed promise (with modest differences in memory and global cognition scores) in slowing the onset of dementia, higher dropout rates in the intervention groups make these results difficult to interpret.

\section{Exercise for people with dementia}

Studies of exercise interventions for patients with dementia have not consistently demonstrated significant improvement in cognition, although they do consistently show improvement in physical performance, ${ }^{79}$ behavior, ${ }^{80}$ and mood. ${ }^{80,81}$ Reviews by Blankevoort et $\mathrm{al}^{82}$ and Forbes et $\mathrm{al}^{83}$ failed to show an effect of exercise interventions on cognitive performance in patients with cognitive impairment. In contrast, an earlier review and meta-analysis of 12 interventions which examined the effects of exercise programs in people with cognitive impairment did demonstrate a moderate effect size (combined effect size 0.57 ) for cognitive performance. ${ }^{84}$ One small clinical trial of a 12-month exercise program for patients with moderate to severe dementia (MMSE 13-14 at baseline) demonstrated a significant improvement in cognition in the exercise group as compared to the control group, but these findings are difficult to interpret as the researchers did not describe how the control group was handled..$^{85}$

\section{Summary: physical exercise and cognitive performance}

Moderate intensity physical exercise can lead to significant changes in brain health and cognitive performance ${ }^{31}$ with potential effects on a broad range of cognitive domains, including memory, attention, and executive function. Starting a moderate intensity physical exercise regimen at any time in later adulthood is beneficial, especially for very sedentary individuals. ${ }^{10,86}$ Although causal mechanisms are unclear, regular exercise likely impacts multiple modifiable risk factors that result in improved brain health and cognitive performance in older adults. ${ }^{87}$

The results of observational studies support a dosedependent neuroprotective relationship between physical exercise and cognitive performance in older adults. This is evident through reduced risk for cognitive decline, MCI, and dementia. Although longitudinal studies offer compelling evidence that higher levels of physical exercise are protective against cognitive decline, questions remain about the effectiveness of interventions. ${ }^{88}$ The results of RCTs of exercise intervention in nondemented older adults have demonstrated improvements that range from no change to moderate gains in global and specific areas of cognitive performance. Equivocal results of exercise RCTs on cognition may be attributed to variability in study design, population characteristics, and exercise parameters. ${ }^{63,64,72}$ Exercise type and intensity differ and some interventions may not be long enough to observe changes in cognitive function. For example, an overall exercise duration of 6-12 months was required in several studies before cognitive improvements were observed. Physical function likely improves prior to cognitive performance gains, providing a stronger foundation from which to progress exercise dosage through increasing intensity, frequency, and duration of exercise.

A duration of at least 16-20 weeks may be needed to sufficiently improve cardiorespiratory fitness in older adults, ${ }^{31}$ and exercise durations of 6-12 months are often needed before cognitive changes are detected. ${ }^{72}$ Therefore, methods to facilitate long-term exercise participation should be incorporated into the design of exercise programs for older adults, such as individually designed programs that address physical limitations, activity preferences, and social needs.

\section{Targeting neuroprotective mechanisms in older adults}

Although exact mechanisms are unclear, it is likely that multiple physiological mechanisms lead to preservation and improvement in brain health and function. ${ }^{38}$ In deriving recommendations for exercise, we make some foundational assumptions about the effects of physical exercise in older adults. First, exercise has neuroprotective effects and reduces risk for disease processes. Second, several physiologic 
mechanisms, including increased glucose sensitivity, neurotrophins, and cerebral perfusion are mediated by exercise and also play a role in neuroprotection. Third, exercise leads to brain plasticity via neurogenesis and synaptogenesis. Potential intermediate outcomes that mediate the effect of physical exercise on cognitive performance include improved overall brain health and brain resilience, increased neurotrophic factors that stimulate neurogenesis, and increased cerebrovascular perfusion. ${ }^{38}$ The metabolic challenge that best produces neuroprotective effects has not been elucidated; however, moderate intensity physical exercise appears to be effective in improving cognitive functions in several studies of aerobic and resistance exercise..$^{73,89}$

\section{Aerobic exercise recommendations}

A positive relationship between aerobic fitness, brain health, and cognitive performance provides a basis for targeting cardiovascular fitness via aerobic exercise interventions. ACSM Guidelines (2009) recommend a minimum of 150 minutes of moderate aerobic exercise per week (30 minutes, 5 days/week) or 60 minutes of vigorousintensity aerobic exercise (20 minutes, 3 days/week). ${ }^{31}$ Intermittent aerobic exercise accrued in 6-10-minute bouts can be effective in improving aerobic capacity, especially for those with limited exercise capacity. ${ }^{90}$ To significantly increase $\mathrm{VO}_{2}$ max in healthy older adults, aerobic exercise intensity should be $\geq 60 \%$ of their pretraining $\mathrm{VO}_{2} \max$ and last at least 16 weeks ${ }^{15,31}$ Larger improvements in $\mathrm{VO}_{2}$ max are typically observed with longer training periods (20-30 weeks) but not necessarily higher training intensities (ie, $>70 \%$ of $\mathrm{VO}_{2}$ max). ${ }^{31,91}$ However, varying aerobic exercise intensity through interval training ( 3 minutes low intensity/3 minutes high intensity) has been shown to be more effective than 60 minutes of steady pace walking in improving $\mathrm{VO}_{2}$ max and glycemic control in people with type 2 diabetes. ${ }^{92}$

\section{Resistance exercise recommendations}

Long-term participation in resistance exercise programs has demonstrated improvements in cognitive performance, especially executive functions. ${ }^{31}$ The ACSM recommends resistance exercises for major muscle groups 2 days/week for older adults. ${ }^{31}$ A resistance training program that emphasizes dynamic exercises involving concentric (shortening) and eccentric (lengthening) muscle actions that includes major muscle groups of the upper and lower body is recommended for all adults. ${ }^{11}$ A resistance equivalent to $60 \%-80 \%$ of an individual's one repetition maximum (1 RM) effort with healthy novices training at $60 \%-70 \%$ of their 1 RM. Older, deconditioned, or frail individuals may begin with lower resistance levels, such as $40 \%-50 \%$ of their 1 RM. ${ }^{11}$ The selected weight should be adjusted so that the individuals can complete 8-12 repetitions/set for strengthening purposes or higher repetitions/set for endurance purposes (eg, 15-20 repetitions at $40 \%-50 \%$ of the $1 \mathrm{RM}$ ). Two to three sets/exercise with rest for 2-3 minutes between each set and a recovery period of 48-72 hours should be allowed between moderate to vigorous intensity exercise sessions. ${ }^{11}$ Neuromuscular fitness (balance, agility, coordination) and flexibility exercises are also recommended 2 days/week to enhance neuromuscular fitness.

\section{Program considerations}

When comparing structured exercise training, consisting of aerobic, resistance, or both, to advice on physical activity, structured training was more effective than advice alone in improving glycemic control in individuals with type 2 diabetes. ${ }^{93}$ Physical exercise programs that are individually designed to accommodate physical and cognitive impairments in older adults may serve to lessen secondary cognitive decline. ${ }^{94}$ Exercise prescription tailored to the individual will improve the potential for maintaining a steady exercise program under safe conditions. Strategies to address cognitive barriers to exercise participation have been recommended by Teri et al. ${ }^{95}$ These include providing exercise instructions in easy-to-remember increments, high repetition and practice, a cognitively intact exercise partner, providing written and visual memory cues, and making the exercise fun and individualized. Further research is needed to establish an evidence base that can support the implementation of exercise for various subgroups of adults with physical and cognitive disabilities. $^{94}$

\section{Conclusion}

Physical exercise that targets modifiable risk factors and neuroprotective mechanisms provides a nonpharmacological approach to slowing age-related decline and reducing disease-related cognitive impairment in older adults. Higher doses of physical exercise are associated with reduced risk for cognitive impairment and dementia. Studies provide compelling evidence that exercise can modify metabolic, structural, and functional dimensions of the brain that preserve cognitive performance in older adults. Further research is needed to identify the most beneficial aspects of exercise programs; however, evidence supports structured, longer duration, and multicomponent exercise programs 
for enhancing cognitive performance and overall function in older adults.

\section{Disclosure}

The authors report no conflicts of interest in this work.

\section{References}

1. United States Census Bureau. US Census Bureau projections show a slower growing, older, more diverse nation a half century from now. 2012. Available at: http://www.census.gov/newsroom/ releases/archives/population/cb12-243.html. Accessed November 2, 2013.

2. Plassman BL, Langa KM, Fisher GG, et al. Prevalence of dementia in the United States: the aging, demographics, and memory study. Neuroepidemiology. 2007;29(1-2):125-132.

3. Plassman BL, Langa KM, Fisher GG, et al. Prevalence of cognitive impairment without dementia in the United States. Ann Intern Med. 2008;148(6):427-434.

4. Jernigan TL, Archibald SL, Fennema-Notestine C, et al. Effects of age on tissues and regions of the cerebrum and cerebellum. Neurobiol Aging. 2001;22(4):581-594.

5. Glisky EL. Changes in cognitive function in human aging. In: Riddle DR, editor. Brain Aging: Models, Methods, and Mechanisms. Boca Raton, FL: CRC Press; 2007:3-20.

6. Dickerson BC, Salat DH, Bates JF, et al. Medial temporal lobe function and structure in mild cognitive impairment. Ann Neurol. 2004;56(1):27-35

7. Dickerson BC, Sperling RA. Functional abnormalities of the medial temporal lobe memory system in mild cognitive impairment and Alzheimer's disease: insights from functional MRI studies. Neuropsychologia. 2008;46(6):1624-1635.

8. Smith CD, Chebrolu H, Wekstein DR, et al. Brain structural alterations before mild cognitive impairment. Neurology. 2007;68(16): 1268-1273.

9. Colcombe SJ, Erickson KI, Scalf PE, et al. Aerobic exercise training increases brain volume in aging humans. J Gerontol A Biol Sci Med Sci. 2006;61(11):1166-1170.

10. Erickson KI, Miller DL, Weinstein AM, Akl SL, Banducci SE. Physical activity and brain plasticity in late adulthood: a conceptual review. Ageing Res. 2012;3(1):34-47.

11. Garber CE, Blissmer B, Deschenes MR, et al; American College of Sports Medicine. American College of Sports Medicine position stand. Quantity and quality of exercise for developing and maintaining cardiorespiratory, musculoskeletal, and neuromotor fitness in apparently healthy adults: guidance for prescribing exercise. Med Sci Sports Exerc. 2011;43(7):1334-1359.

12. Grodstein F. Cardiovascular risk factors and cognitive function. Alzheimers Dement. 2007;3(Suppl 2):S16-S22.

13. Radak Z, Hart N, Sarga L, et al. Exercise plays a preventive role against Alzheimer's disease. J Alzheimers Dis. 2010;20(3):777-783.

14. Intlekofer KA, Cotman CW. Exercise counteracts declining hippocampal function in aging and Alzheimer's disease. Neurobiol Dis. 2013;57:47-55.

15. Nelson ME, Rejeski WJ, Blair SN, et al; American College of Sports Medicine; American Heart Association. Physical activity and public health in older adults: recommendation from the American College of Sports Medicine and the American Heart Association. Circulation. 2007;116(9):1094-1105.

16. Knecht $\mathrm{S}$, Wersching $\mathrm{H}$, Lohmann $\mathrm{H}$, et al. High-normal blood pressure is associated with poor cognitive performance. Hypertension. 2008;51(3):663-668.

17. Kivipelto M, Ngandu T, Fratiglioni L, et al. Obesity and vascular risk factors at midlife and the risk of dementia and Alzheimer disease. Arch Neurol. 2005;62(10):1556-1560.
18. Tervo S, Kivipelto M, Hänninen $T$, et al. Incidence and risk factors for mild cognitive impairment: a population-based three-year follow-up study of cognitively healthy elderly subjects. Dement Geriatr Cogn Disord. 2004;17(3):196-203.

19. Kivipelto M, Helkala EL, Laakso MP, et al. Midlife vascular risk factors and Alzheimer's disease in later life: longitudinal, population based study. BMJ. 2001;322(7300):1447-1451.

20. Fujishima M, Ibayashi S, Fujii K, Mori S. Cerebral blood flow and brain function in hypertension. Hypertens Res. 1995;18(2):111-117.

21. Jennings JR, Muldoon MF, Ryan CM, et al. Cerebral blood flow in hypertensive patients: an initial report of reduced and compensatory blood flow responses during performance of two cognitive tasks. Hypertension. 1998;31(6):1216-1222.

22. Waldstein SR. The relation of hypertension to cognitive function. Curr Dir Psychol Sci. 2003;12(1):9-12.

23. Sparks DL, Scheff SW, Liu H, Landers TM, Coyne CM, Hunsaker JC. Increased incidence of neurofibrillary tangles (NFT) in non-demented individuals with hypertension. J Neurol Sci. 1995;131(2):162-169.

24. Peila R, White LR, Masaki K, Petrovitch H, Launer LJ. Reducing the risk of dementia: efficacy of long-term treatment of hypertension. Stroke. 2006;37(5):1165-1170.

25. Yaffe K, Kanaya A, Lindquist K, et al. The metabolic syndrome, inflammation, and risk of cognitive decline. JAMA. 2004;292(18): 2237-2242.

26. Yaffe K, Lindquist K, Penninx BW, et al. Inflammatory markers and cognition in well-functioning African-American and white elders. Neurology. 2003;61(1):76-80.

27. Colbert LH, Visser M, Simonsick EM, et al. Physical activity, exercise, and inflammatory markers in older adults: findings from the Health, Aging and Body Composition Study. J Am Geriatr Soc. 2004;52(7):1098-1104.

28. Vincent KR, Braith RW, Bottiglieri T, Vincent HK, Lowenthal DT. Homocysteine and lipoprotein levels following resistance training in older adults. Prev Cardiol. 2003;6(4):197-203.

29. Craft S, Cholerton B, Baker LD. Insulin and Alzheimer's disease: untangling the web. J Alzheimers Dis. 2013;33 Suppl 1:S263-S275.

30. Baker LD, Frank LL, Foster-Schubert K, et al. Aerobic exercise improves cognition for older adults with glucose intolerance, a risk factor for Alzheimer's disease. J Alzheimers Dis. 2010;22(2):569-579.

31. Chodzko-Zajko WJ, Proctor DN, Fiatarone Singh MA, et al; American College of Sports Medicine. American College of Sports Medicine position stand. Exercise and physical activity for older adults. Med Sci Sports Exerc. 2009;41(7):1510-1530.

32. Vaynman S, Gomez-Pinilla F. License to run: exercise impacts functional plasticity in the intact and injured central nervous system by using neurotrophins. Neurorehabil Neural Repair. 2005;19(4):283-295.

33. Rasmussen P, Brassard P, Adser H, et al. Evidence for a release of brain-derived neurotrophic factor from the brain during exercise. Exp Physiol. 2009;94(10):1062-1069.

34. Seifert T, Brassard P, Wissenberg M, et al. Endurance training enhances BDNF release from the human brain. Am J Physiol Regul Integr Comp Physiol. 2010;298(2):R372-R377.

35. Cotman CW, Berchtold NC. Exercise: A behavioral intervention to enhance brain health and plasticity. Trends Neurosci. 2002;25(6): 295-301.

36. Cassilhas RC, Viana VA, Grassmann V, et al. The impact of resistance exercise on the cognitive function of the elderly. Med Sci Sports Exerc. 2007;39(8):1401-1407.

37. Ahlskog JE, Geda YE, Graff-Radford NR, Petersen RC. Physical exercise as a preventive or disease-modifying treatment of dementia and brain aging. Mayo Clin Proc. 2011;86(9):876-884.

38. Cotman CW, Berchtold NC. Physical activity and the maintenance of cognition: learning from animal models. Alzheimers Dement. 2007;3(Supp1 2):S30-S37.

39. Adlard A, Perreau VM, Pop V, Cotman CW. Voluntary exercise decreases amyloid load in a transgenic model of Alzheimer's disease. J Neurosci. 2005;25(17):4217-4221. 
40. van Praag H, Shubert T, Zhao C, Gage FH. Exercise enhances learning and hippocampal neurogenesis in aged mice. $J$ Neurosci. 2005;25(38):8680-8685.

41. Swain RA, Harris AB, Wiener EC, et al. Prolonged exercise induces angiogenesis and increases cerebral blood volume in primary motor cortex of the rat. Neuroscience. 2003;117(4):1037-1046.

42. Black JE, Isaacs KR, Anderson BJ, Alcantara AA, Greenough WT. Learning causes synaptogenesis, whereas motor activity causes angiogenesis, in cerebellar cortex of adult rats. Proc Natl Acad Sci US A. 1990;87(14):5568-5572.

43. Angevaren M, Aufdemkampe G, Verhaar HJJ, Aleman A, Vanhees L. Physical activity and enhanced fitness to improve cognitive function in older people without known cognitive impairment. Cochrane Database of Syst Rev. 2008;3.

44. Burns JM, Cronk BB, Anderson HS, et al. Cardiorespiratory fitness and brain atrophy in early Alzheimer disease. Neurology. 2008;71(3): 210-216.

45. Honea RA, Thomas GP, Harsha A, et al. Cardiorespiratory fitness and preserved medial temporal lobe volume in Alzheimer disease. Alzheimer Dis Assoc Disord. 2009;23(3):188-197.

46. Colcombe SJ, Erickson KI, Raz N, et al. Aerobic fitness reduces brain tissue loss in aging humans. J Gerontol A Biol Sci Med Sci. 2003;58(2):176-180.

47. Erickson KI, Prakash RS, Voss MW, et al. Aerobic fitness is associated with hippocampal volume in elderly humans. Hippocampus. 2009;19(10):1030-1039.

48. Colcombe SJ, Kramer AF, Erickson KI, et al. Cardiovascular fitness, cortical plasticity, and aging. Proc Natl Acad Sci U S A. 2004;101(9) 3316-3321.

49. Voss MW, Erickson KI, Prakash RS, et al. Functional connectivity: A source of variance in the association between cardiorespiratory fitness and cognition? Neuropsychologia. 2010;48(5):1394-1406.

50. Weuve J, Kang JH, Manson JE, Breteler MM, Ware JH, Grodstein F. Physical activity, including walking, and cognitive function in older women. JAMA. 2004;292(12):1454-1461.

51. van Gelder BM, Tijhuis MA, Kalmijn S, Giampaoli S, Nissinen A, Kromhout D. Physical activity in relation to cognitive decline in elderly men: the FINE Study. Neurology. 2004;63(12):2316-2321.

52. Abbott RD, White LR, Ross GW, Masaki KH, Curb JD, Petrovitch H. Walking and dementia in physically capable elderly men. JAMA 2004;292(12):1447-1453.

53. Taaffe DR, Irie F, Masaki KH, et al. Physical activity, physical function, and incident dementia in elderly men: the Honolulu-Asia Aging Study. J Gerontol A Biol Sci Med Sci. 2008;63(5): 529-535.

54. Yaffe K, Barnes D, Nevitt M, Lui LY, Covinsky K. A prospective study of physical activity and cognitive decline in elderly women: women who walk. Arch Intern Med. 2001;161(14):1703-1708.

55. Hamer M, Chida Y. Physical activity and risk of neurodegenerative disease: a systematic review of prospective evidence. Psychol Med. 2009;39(1):3-11.

56. Bugg JM, Head D. Exercise moderates age-related atrophy of the medial temporal lobe. Neurobiol Aging. 2011;32(3):506-514.

57. Erickson KI, Raji CA, Lopez OL, et al. Physical activity predicts gray matter volume in late adulthood: the Cardiovascular Health Study. Neurology. 2010;75(16):1415-1422.

58. Angevaren M, Vanhees L, Wendel-Vos W, et al. Intensity, but not duration, of physical activities is related to cognitive function. Eur $J$ Cardiovasc Prev Rehabil. 2007;14:825-830.

59. Podewils LJ, Guallar E, Kuller LH, et al. Physical activity, APOE genotype, and dementia risk: findings from the Cardiovascular Health Cognition Study. Am J Epidemiol. 2005;161(7): 639-651.

60. Bixby WR, Spalding TW, Haufler AJ, et al. The unique relation of physical activity to executive function in older men and women. Med Sci Sports Exerc. 2007;39(8):1408-1416.

61. Geda Y, Roberts RO, Knopman DS, et al. Physical exercise, aging, and mild cognitive impairment. Arch Neurol. 2010;67:80-86.
62. Smith PJ, Blumenthal JA, Hoffman BM, et al. Aerobic exercise and neurocognitive performance: a meta-analytic review of randomized controlled trials. Psychosom Med. 2010;72(3):239-252.

63. Snowden M, Steinman L, Mochan K, et al. Effect of exercise on cognitive performance in community-dwelling older adults: review of intervention trials and recommendations for public health practice and research. J Am Geriatr Soc. 2011;59(4):704-716.

64. van Uffelen JG, Chin A, Paw MJ, Hopman-Rock M, van Mechelen W. The effects of exercise on cognition in older adults with and without cognitive decline: A systematic review. Clin J Sport Med. 2008;18(6):486-500.

65. Etnier JL, Nowell PM, Landers DM, Sibley BA. A meta-regression to examine the relationship between aerobic fitness and cognitive performance. Brain Res Rev. 2006;52(1):119-130.

66. Erickson KI, Voss MW, Prakash RS, et al. Exercise training increases size of hippocampus and improves memory. Proc Natl Acad Sci U S A. 2011;108(7):3017-3022.

67. Voss MW, Prakash RS, Erickson KI, et al. Plasticity of brain networks in a randomized intervention trial of exercise training in older adults. Front Aging Neurosci. 2010;2:1-17.

68. Voss MW, Erickson KI, Prakash RS, et al. Neurobiological markers of exercise-related brain plasticity in older adults. Brain Behav Immun. 2013;28:90-99.

69. Lautenschlager NT, Cox KL, Flicker L, et al. Effect of physical activity on cognitive function in older adults at risk for Alzheimer disease: a randomized trial. JAMA. 2008;300(9):1027-1037.

70. Baker LD, Frank LL, Foster-Schubert K, et al. Effects of aerobic exercise on mild cognitive impairment: a controlled trial. Arch Neurol. 2010;67(1):71-79.

71. Suzuki T, Shimada H, Makizako H, et al. Effects of multicomponent exercise on cognitive function in older adults with amnestic mild cognitive impairment: a randomized controlled trial. BMC Neurol. $2012 ; 12: 128$.

72. Lautenschlager NT, Cox KL. Can participation in mental and physical activity protect cognition in old age?: Comment on "The Mental Activity and eXercise (MAX) trial: a randomized controlled trial to enhance cognitive function in older adults". JAMA Intern Med. 2013;173(9):805-806

73. Liu-Ambrose T, Nagamatsu LS, Graf P, Beattie BL, Ashe MC, Handy TC. Resistance training and executive functions: a 12-month randomized controlled trial. Arch Intern Med. 2010;170(2): $170-178$.

74. Liu-Ambrose T, Nagamatsu LS, Voss MW, Khan KM, Handy TC. Resistance training and functional plasticity of the aging brain: a 12-month randomized controlled trial. Neurobiol Aging. 2012;33(8): 1690-1698.

75. Liu-Ambrose T, Donaldson MG, Ahamed Y, et al. Otago home-based strength and balance retraining improves executive functioning in older fallers: a randomized controlled trial. J Am Geriatr Soc. 2008;56(10):1821-1830.

76. Chang YK, Nien YH, Tsai CL, Etnier JL. Physical activity and cognition in older adults: the potential of Tai Chi Chuan. J Aging Phys Act. 2010;18(4):451-472.

77. Chan AS, Ho YC, Cheung MC, Albert MS, Chiu HF, Lam LC. Association between mind-body and cardiovascular exercises and memory in older adults. J Am Geratr Soc. 2005;53(10):1754-1760.

78. Lam LC, Chau RC, Wong BM, et al. A 1-year randomized controlled trial comparing mind body exercise (Tai Chi) with stretching and toning exercise on cognitive function in older Chinese adults at risk of cognitive decline. J Am Med Dir Assoc. 2012;13:568.e15-568.e20.

79. Roach KE, Tappen RM, Kirk-Sanchez N, Williams CL, Loewenstein DL. A randomized controlled trial of an activity-specific exercise program for individuals with Alzheimer's disease in long-term care settings. J Geriatr Phys Ther. 2011;34(2):50-56.

80. Teri L, Gibbons LE, McCurry SM, et al. Exercise plus behavioral management in patients with Alzheimer disease: a randomized controlled trial. JAMA. 2003;290(15):2015-2022. 
81. Williams CL, Tappen RM. Effect of exercise on mood in nursing home residents with Alzheimer's disease. Am J Alzheimers Dis Other Demen. 2007;22(5):389-397.

82. Blankevoort CG, van Heuvelen MJ, Boersma F, Luning H, de Jong J, Scherder EJ. Review of effects of physical activity on strength, balance, mobility and ADL performance in elderly subjects with dementia. Dement Geriatr Cogn Disord. 2010;30(5):392-402.

83. Forbes D, Forbes S, Morgan DG, Markle-Reid M, Wood J, Culum I. Physical activity programs for persons with dementia (Review). Cochrane Database Syst Rev. 2008;16(3):CD006489.

84. Heyn P, Abreu BC, Ottenbacher KJ. The effects of exercise training on elderly persons with cognitive impairment and dementia: a metaanalysis. Arch Phys Med Rehabil. 2004;85(10):1694-1704.

85. Kwak YS, Um SY, Son TG, Kim DJ. Effect of regular exercise on senile dementia patients. Int J Sports Med. 2008;29(6):471-474.

86. Middleton LE, Yaffe K. Targets for the prevention of dementia. J Alzheimers Dis. 2010;20(3):915-924.

87. Fotuhi M, Do D, Jack C. Modifiable factors that alter the size of the hippocampus with ageing. Nat Rev Neurol. 2012;8(4):189-202.

88. Larson EB. Physical activity for older adults at risk for Alzheimer disease. JAMA. 2008;300(9):1077-1079.
89. Colcombe S, Kramer AF. Fitness effects on the cognitive function of older adults: A meta-analytic study. Psychol Sci. 2003;14(2):125-130.

90. Macfarlane DJ, Taylor LH, Cuddihy TF. Very short intermittent vs continuous bouts of activity in sedentary adults. Prev Med. 2006;43(4): 332-336.

91. Huang G, Gibson CA, Tran ZV, Osness WH. Controlled endurance exercise training and $\mathrm{VO} 2$ max changes in older adults: A meta-analysis. Prev Cardiol. 2005;8(4):217-225.

92. Karstoft K, Winding K, Knudsen SH, et al. The effects of free-living interval-walking training on glycemic control, body composition, and physical fitness in type 2 diabetic patients: a randomized, controlled trial. Diabetes Care. 2013;36(2):228-236.

93. Umpierre D, Ribeiro PA, Kramer CK, et al. Physical activity advice only or structured exercise training and association with $\mathrm{HbA} 1 \mathrm{c}$ levels in type 2 diabetes: a systematic review and meta-analysis. JAMA. 2011;305(17):1790-1799.

94. Rimmer JH, Chen MD, McCubbin JA, Drum C, Peterson J. Exercise intervention research on persons with disabilities: what we know and where we need to go. Am J Phys Med Rehabil. 2010;89(3):249-263.

95. Teri L, Logsdon RG, McCurry SM. Exercise interventions for dementia and cognitive impairment: the Seattle Protocols. J Nutr Health Aging. 2008;12(6):391-394.-
Clinical Interventions in Aging

\section{Publish your work in this journal}

Clinical Interventions in Aging is an international, peer-reviewed journal focusing on evidence-based reports on the value or lack thereof of treatments intended to prevent or delay the onset of maladaptive correlates of aging in human beings. This journal is indexed on PubMed Central, MedLine, the American Chemical Society's 'Chemical Abstracts

\section{Dovepress}

Service' (CAS), Scopus and the Elsevier Bibliographic databases. The manuscript management system is completely online and includes a very quick and fair peer-review system, which is all easy to use. Visit $\mathrm{http}: / /$ www.dovepress.com/testimonials.php to read real quotes from published authors. 